\title{
Checklist of Nematodes (Nematoda: Adenophorea) from Southeast Continental Shelf of India
}

\author{
Kapuli Gani Mohamed Thameemul Ansari*, Seerangan Manokaran, Sanjeevi Raja, Syed Ajmal Khan \\ and Somasundharanair Lyla
}

Annamalai University, Faculty of marine Sciences, Centre of Advanced Study in Marine biology. Parangipettai - 608 502, Tamil Nadu, India.

* Corresponding author. E-mail: casthameem@gmail.com

\begin{abstract}
A checklist of free-living marine nematode species recorded from the continental shelf region of southeast coast of India is presented $\left(10^{\circ} 34.03^{\prime}\right.$ to $15^{\circ} 14.48^{\prime} \mathrm{N}$ and from $79^{\circ} 52.13^{\prime}$ to $\left.80^{\circ} 53.87^{\prime} \mathrm{E}\right)$. The checklist comprise 191 species belonging to 97 genera in 32 families currently know from the area. We provided a synthesis of the taxonomical and ecological work carried out until now that could guide to future research
\end{abstract}

\section{INTRODUCTION}

Sedimentary habitats cover most of the ocean bottom and therefore constitute the largest single ecosystem on earth in spatial coverage (Schratzberger et al. 2007). Benthic organisms which occupy these habitats make a significant contribution to the regulation of carbon, nitrogen and sulphur cycling, water column process, pollutant distribution and fate, secondary production and transport and stability of sediments (Snelgrove et al. 1997). Four of every five bottom living multicellular animals on earth are nematodes (Bongers and Ferries 1999). Free living marine nematodes are usually the most abundant metazoans inhabiting marine benthic ecosystems, often representing more than $60-90 \%$ of the benthic meiofauna (Sajan et al. 2010).

Their significance in terms of energy flexes in the food chain through way of degradation and mineralization of organic matter is high. Nematodes have short life span, high fecundity (Vranken and Heip 1983), represent several tropic levels (herbivores, bacterial feeders and carnivores) and at least some species can be easily cultured (Heip et al. 1985). By virtue of their wide range of adaptations, marine nematodes have exploited all seashores and seabed habitats. An important feature of nematode population is the large number of species present in any habitat, often an order of magnitude higher than for any other taxon (Platt and Warwick 1980).

Whereas the importance of parasitic nematodes has been recognised for many decades, this is not the case for free-living marine nematode species, especially those of aquatic environments. They remain poorly understood, despite the fact that they are extremely abundant and diverse, often numbering millions per square meter in sediments, and occur in more habitats than any other metazoan group (Heip et al. 1985). While few studies have been carried out on nematodes in and around the Indian waters, nematode communities of the Indian shelf sediments received cursory attention. More over the nematofaunal studies done in the Indian subcontinent covered mostly the Western continental shelf (Harkantra et al. 1980; Sajan et al. 2010) and shallow coastal waters (Timm, 1961; 1967; Ansari et al. 1980) and no information is available on the distribution and species level composition of nematodes in the Southeast coast of India. Therefore the present investigation was undertaken on the meiobenthos especially on nematodes in the continental shelf of the Southeast coast of India.

\section{Materials AND Methods \\ Study site}

The study area extends from $10^{\circ} 34.03^{\prime}$ to $15^{\circ} 14.48^{\prime}$ $\mathrm{N}$ and from $79^{\circ} 52.13^{\prime}$ to $80^{\circ} 53.87^{\prime} \mathrm{E}$ in the continental shelf region of the Southeast coast of India (Figure 1). Totally 35 sediment samples were collected along 6 transects (off Karaikkal, Parangipettai, Cheyyur, Chennai, Tammenapatanam and Singarayakonda) at the depths of $30-50 \mathrm{~m}, 50-75 \mathrm{~m}, 75-100 \mathrm{~m}, 100-150 \mathrm{~m}, 150-175 \mathrm{~m}$ and above $175 \mathrm{~m}$. In addition one more transect was sampled, due to the presence of an industrial cluster in CuddaloreSIPCOT (State Industries Promotion Corporation of Tamil $\mathrm{Nadu}$ ), at $30-50 \mathrm{~m}$ and above $175 \mathrm{~m}$ depths. In these industrial cluster units mainly produced PVC pipes, paints, pharmaceutical products and fertilizers. These industries play a major role in this region and the industrial units here discharge treated/untreated effluents in to the sea. The study area map was drawn with the help of SURFER 8.0.

\section{Data collection}

Meiofaunal samples for the present study were collected onboard FORV (Fishery and Oceanographic Research Vessel) "Sagar Sampada" during Cruise No. 260 under the program of "Marine Benthos of Indian EEZ" founded by Centre for Marine Living Resources and Ecology (CMLRE), Ministry of Earth Sciences (MoES), Government of India. Two samples were collected using a 
Smith McIntyre grab (having a bite area of $0.2 \mathrm{~m}^{2}$ ) from each depth. Immediately after the grab was hauled to the deck and sub-samples were taken from undisturbed grab samples using a glass corer (with an internal diameter of $2.5 \mathrm{~cm}$ and a length of $15 \mathrm{~cm}$ ) from the middle of grab sample (Platt and Warwick 1983). The samples were fixed in buffered formalin at a concentration of $4 \%$. The replicate core samples were processed separately in the laboratory and data were pooled for analyses.

\section{Sample processing}

In the laboratory, samples were washed through a set of $0.5 \mathrm{~mm}$ and $0.063 \mathrm{~mm}$ sieves. The sediment retained in the $0.063 \mathrm{~mm}$ sieve was decanted to extract meiofauna following the method (Higgins and Thiel, 1988). Sorting of meiofauna from sediment was done by flotation technique. The efficiency of this technique is around 95\% followed by Armenteros et al. (2008). The meiofaunal organisms were stained with Rose Bengal prior to extraction and were sorted and enumerated under a stereomicroscope (Meiji, Japan). All the nematodes were mounted onto glass slides, using the formalin-ethanol-glycerol technique by Vincx (1996). Identification of nematodes was carried out to the highest taxonomic level possible using the compound microscope (Olympus CX 41 under higher magnification of 1000x) following the standard pictorial keys of Platt and Warwick (1983; 1988), Warwick et al. (1998) and the NeMys Database (Steyaert et al. 2005).

The collected specimens is currently deposited in CMLRE (Centre for Marine Living Resource and Ecology), MoES (Ministry of Earth Sciences), Government of India, whose available voucher numbers are provided (Table 1). These lists could be found in "Achievements of FORV Sagar Sampada 1997-2010" published in National Symposium entitled "Indian Ocean Marine Living Resources

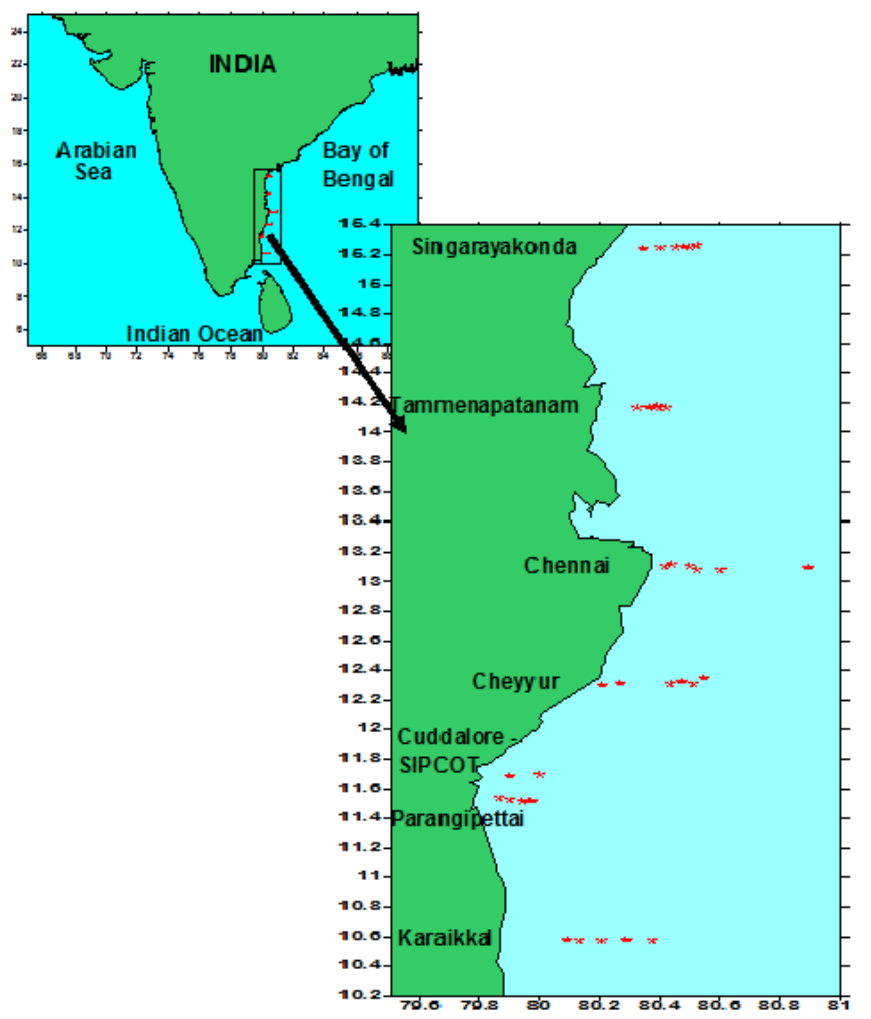

FIGURE 1. Depths sampled at various transects of southeast continental shelf of India.
(IndoMLR)", held at CMLRE, Kochi, India 2010.

\section{RESULTS AND DisCUSSION}

A total of 4218 individuals were collected and 191 species belonging to 97 genera and 32 families were identified. About 154 species of nematodes were reported from the Western continental shelf of India (Sajan et al. 2010). In the present study, the abundance (mean \pm SE) of nematodes decreased with increase in depth. The maximum abundance of $207.7 \pm 19.19$ no. of ind. $/ 10 \mathrm{~cm}^{2}$ was noticed in $30-50 \mathrm{~m}$ depth and the minimum in $>175 \mathrm{~m}$ depth $\left(34.6 \pm 6.66\right.$ no. of ind. $\left./ 10 \mathrm{~cm}^{2}\right)$. Highest number of species was recorded in $30-50 \mathrm{~m}$ depth (151 species) followed by $100-150 \mathrm{~m}$ depth (120 species), 75-100m depth(100 species), 50-75m depth (95 species) , 150$175 \mathrm{~m}$ depth (80 species) and $>175 \mathrm{~m}$ depth(58 species) (Table 1). As observed in the present study, decline in abundance, number of species and families with increase in depth was reported (Ansari et al. 1980; Parulekar et al. 1982; Muthumbi et al. 2004; Sajan and Damodaran 2007; Sajan et al. 2010) in the Indian shelf sediments and (De Bovee et al. 1990; Tietjen 1992; Soltwedel 2000; Liu et al. 2007; De Leonardis et al. 2008; Armenteros et al. 2009) from other parts of the world. Transect-wise variation was less distinct in the faunal composition. However, such a variation was noticed in the abundance of nematodes in the entire shelf (Sajan et al. 2010).

Seventeen families such as Anoplostomatidae, Oxystominidae, Oncholaimidae, Trefusiidae, Chromadoridae, Comesomatidae, Cyatholaimidae, Desmodoridae, Microlaimidae, Leptolaimidae, Ceramonematidae, Desmoscolecidae, Monhysteridae, Xyalidae, Sphaerolaimidae, Linhomidae and Axonolaimidae were found in the entire study area. Some families were found to be depth specific- Enchelidiidae and Epsilonematidae were restricted only in $30-50 \mathrm{~m}$ depth range, Meyliidae in 50-75m depth and Enoplidae in 75- $100 \mathrm{~m}$ depth. Totally 29 out of 32 nematode families were recorded in $30-50 \mathrm{~m}$ depth followed by 27 families in $50-75 \mathrm{~m}$ and $100-150 \mathrm{~m}$ depths. While behind $100 \mathrm{~m}$ depth all the 32 nematode families were found and beyond $100 \mathrm{~m}$ depth 28 families were recorded. However Enoplidae, Enchelidiidae, Epsilonematidae and Meyliidae were not recorded. At the edge of the shelf (beyond 175m) only 18 families were recorded. Ironidae, Leptosomatidae and Draconematidae were present in all the depth ranges except $>175 \mathrm{~m}$ depth (Table 1). Similar results were reported by Sajan and Damodaran (2007) in Western continental shelf of India.

The present study most of the free-living marine nematodes were identified only generic level for the reason that lack of literature. The generic composition in the southeast continental shelf of India showed the presence of all the depths genera like Anoplostoma, Halalaimus, Oxystomina, Viscosia, Dorylaimopsis, Sabatieria, Paralongicyatholaimus, Tricoma, Thalassomonhystera, Daptonema, Terschellingia and Odontophora (Table 1) as found in various shelf sediments (Vanreusel et al. 1992; Soetaert and Heip 1995; Muthumbi et al. 2004; Sajan and Damodaran 2007; Sajan et al. 2010). In the importance of industrial cluster transect (Cuddalore - SIPCOT), nematode species like Mesocanthion sp., Neochromadora 
sp. and Epsilonema pustulatum were recorded only in this transect. Genus Neochromadora was noticed as indicators for oil pollution and heavy metal contamination (Platt and Warwick 1988). There is no information regarding list of free-living marine nematode species, distribution, diversity and pollution aspect studies in the southeast continental shelf of India. Therefore, the present study data is valuable for further ecological, qualitative and quantitative research on free-living marine nematodes in this region.

TABLE 1. List of species collected at seven transects and each in six depths $(1-30-50 \mathrm{~m}, 2=50-75 \mathrm{~m}, 3=75-100 \mathrm{~m}, 4=100-150 \mathrm{~m}, 5=150-175 \mathrm{~m}$ and 6 $=>175 \mathrm{~m}$ ) along the continental shelf region of the southeast coast of India ( $\mathrm{x}-$ present, - absent).

\begin{tabular}{|c|c|c|c|c|c|c|c|}
\hline NEMATODES & CMLRE NUMBER & 1 & 2 & 3 & 4 & 5 & 6 \\
\hline \multicolumn{8}{|l|}{ Enoplidae Dujardin, 1845 (1) } \\
\hline Enoplus sp. & - & - & - & $\mathrm{x}$ & - & - & - \\
\hline \multicolumn{8}{|l|}{ Thoracostomopsidae Filipjev, 1927 (4) } \\
\hline Enoplolaimus longicaudatus (Southern, 1914) & CMLRE 4/061 & - & - & - & - & $\mathrm{x}$ & - \\
\hline Mesacanthion sp. & - & $\mathrm{x}$ & - & - & - & - & - \\
\hline Paramesacanthion sp. & CMLRE 4/068 & $\mathrm{x}$ & $\mathrm{x}$ & - & $\mathrm{x}$ & - & - \\
\hline Thoracostomopsis sp. & - & - & - & - & $\mathrm{x}$ & - & - \\
\hline \multicolumn{8}{|l|}{ Anoplostomatidae Gerlach and Riemann, 1974 (2) } \\
\hline Anoplostoma viviparam (Bastian, 1865) & - & $\mathrm{x}$ & $\mathrm{x}$ & $\mathrm{x}$ & $\mathrm{x}$ & $\mathrm{x}$ & $\mathrm{x}$ \\
\hline Anoplostoma sp. & - & $\mathrm{x}$ & $\mathrm{x}$ & $\mathrm{x}$ & $\mathrm{x}$ & $\mathrm{x}$ & $\mathrm{x}$ \\
\hline \multicolumn{8}{|l|}{ Anticomidae Filipjev, 1918 (2) } \\
\hline Anticoma eberthi Bastian, 1865 & CMLRE 4/006 & - & - & - & $\mathrm{x}$ & - & - \\
\hline Anticoma sp. & - & $\mathrm{x}$ & $\mathrm{x}$ & $\mathrm{x}$ & $\mathrm{x}$ & - & - \\
\hline \multicolumn{8}{|l|}{ Ironidae De Man, 1876 (2) } \\
\hline Dolicholaimus sp. & - & $x$ & - & - & $\mathrm{x}$ & - & - \\
\hline Syringolaimus sp. & - & $\mathrm{x}$ & $\mathrm{x}$ & $\mathrm{x}$ & $\mathrm{x}$ & $\mathrm{x}$ & - \\
\hline \multicolumn{8}{|l|}{ Leptosomatidae Filipjev, 1916 (3) } \\
\hline Leptosomatum sp. & - & $\mathrm{x}$ & - & $\mathrm{x}$ & - & - & - \\
\hline Platycoma sp. & - & $\mathrm{x}$ & $\mathrm{x}$ & - & $\mathrm{x}$ & - & - \\
\hline Thoracostoma sp. & - & $\mathrm{x}$ & - & - & - & $\mathrm{x}$ & - \\
\hline \multicolumn{8}{|l|}{ Oxystominidae Chitwood, 1935 (7) } \\
\hline Halalaimus capitulates Boucher, 1977 & - & $\mathrm{x}$ & - & - & - & - & - \\
\hline Halalaimus gracilis De man, 1888 & - & $\mathrm{x}$ & $\mathrm{x}$ & $\mathrm{x}$ & $\mathrm{x}$ & $\mathrm{x}$ & $\mathrm{x}$ \\
\hline Halalaimus longicaudatus (Filipjev, 1927) & - & $\mathrm{x}$ & $\mathrm{x}$ & $\mathrm{x}$ & $\mathrm{x}$ & $\mathrm{x}$ & $\mathrm{x}$ \\
\hline Halalaimus sp. & - & $\mathrm{x}$ & $\mathrm{x}$ & $\mathrm{x}$ & $\mathrm{x}$ & $\mathrm{x}$ & $\mathrm{x}$ \\
\hline Nemanema sp. & - & - & $\mathrm{x}$ & - & - & - & $\mathrm{x}$ \\
\hline Oxystomina elongata (Butschili, 1874) & - & $\mathrm{x}$ & $\mathrm{x}$ & $\mathrm{x}$ & $\mathrm{x}$ & $\mathrm{x}$ & $\mathrm{x}$ \\
\hline Oxystomina sp. & CMLRE 4/060 & $\mathrm{x}$ & $\mathrm{x}$ & $\mathrm{x}$ & $\mathrm{x}$ & $\mathrm{x}$ & $\mathrm{x}$ \\
\hline \multicolumn{8}{|l|}{ Oncholaimidae Filipjev, 1916 (9) } \\
\hline Metoncholaimus scanicus (Allgen, 1935) & CMLRE $4 / 039$ and $4 / 78$ & $\mathrm{x}$ & - & - & - & - & - \\
\hline Oncholaimellus calvadosicus De Man, 1890 & CMLRE $4 / 057$ & - & - & - & $\mathrm{x}$ & - & - \\
\hline Oncholaimellus sp. & - & - & $\mathrm{x}$ & - & $\mathrm{x}$ & $\mathrm{x}$ & $\mathrm{x}$ \\
\hline Viscosia abyssorum (Allgen, 1933) & - & $\mathrm{x}$ & - & - & - & - & - \\
\hline Viscosia elegans (Kreis, 1924) & - & $\mathrm{x}$ & $\mathrm{x}$ & $\mathrm{x}$ & $\mathrm{x}$ & $\mathrm{x}$ & $\mathrm{x}$ \\
\hline Viscosia glabra (Bastian, 1865) & CMLRE 4/038 & $\mathrm{x}$ & - & $\mathrm{x}$ & $\mathrm{x}$ & $\mathrm{x}$ & - \\
\hline Viscosia langrunensis (De Man, 1890) & CMLRE 4/037 & $x$ & $\mathrm{x}$ & $\mathrm{x}$ & $\mathrm{x}$ & $\mathrm{x}$ & $\mathrm{x}$ \\
\hline Viscosia viscosa (Bastian, 1865) & - & $\mathrm{x}$ & $\mathrm{x}$ & $\mathrm{x}$ & $\mathrm{x}$ & $\mathrm{x}$ & $\mathrm{x}$ \\
\hline Viscosia sp. & - & $\mathrm{x}$ & $\mathrm{x}$ & $\mathrm{x}$ & $\mathrm{x}$ & $\mathrm{x}$ & $\mathrm{x}$ \\
\hline \multicolumn{8}{|l|}{ Enchelidiidae Filipjev, 1918 (2) } \\
\hline Belbolla sp. & CMLRE 4/076 & $\mathrm{x}$ & - & - & - & - & - \\
\hline Pareurystomina sp. & CMLRE 4/058 & $\mathrm{x}$ & $\mathrm{x}$ & - & $\mathrm{x}$ & $\mathrm{x}$ & - \\
\hline \multicolumn{8}{|l|}{ Tripyloididae Filipjev, 1918 (2) } \\
\hline Tripyloides marinus (Butschli, 1874) & - & $\mathrm{x}$ & - & - & $\mathrm{x}$ & - & - \\
\hline Tripyloides sp. & CMLRE 4/059 & $\mathrm{x}$ & - & - & - & - & - \\
\hline \multicolumn{8}{|l|}{ Trefusiidae Gerlach, 1966 (4) } \\
\hline Halanonchus sp. & - & $\mathrm{x}$ & $\mathrm{x}$ & $\mathrm{x}$ & $\mathrm{x}$ & $\mathrm{x}$ & $\mathrm{x}$ \\
\hline Trefusia longicaudata De Man, 1893 & - & $\mathrm{x}$ & $\mathrm{x}$ & $\mathrm{x}$ & $\mathrm{x}$ & - & - \\
\hline Trefusia sp.1 & - & $\mathrm{x}$ & $\mathrm{x}$ & $\mathrm{x}$ & $\mathrm{x}$ & - & - \\
\hline Trefusia sp.2 & - & $\mathrm{x}$ & $\mathrm{x}$ & - & $\mathrm{x}$ & - & - \\
\hline \multicolumn{8}{|l|}{ Chromadoridae Filipjev, 1917 (5) } \\
\hline Chromadora sp. & - & $\mathrm{x}$ & $\mathrm{x}$ & $\mathrm{x}$ & - & - & - \\
\hline Chromadorina granulopigmentata (Weiser, 1951) & CMLRE 4/013 & $\mathrm{x}$ & $\mathrm{x}$ & - & $\mathrm{x}$ & - & - \\
\hline Neochromadora poecilosomoides (Filipjev, 1918) & CMLRE 4/079 & $\mathrm{x}$ & - & - & - & - & - \\
\hline
\end{tabular}


TABle 1. Continued.

\begin{tabular}{|c|c|c|c|c|c|c|c|}
\hline NEMATODES & CMLRE NUMBER & 1 & 2 & 3 & 4 & 5 & 6 \\
\hline Prochromadorella sp. & - & - & - & - & - & - & $\mathrm{x}$ \\
\hline Siplophorella sp. & - & $\mathrm{x}$ & $\mathrm{x}$ & $\mathrm{x}$ & $\mathrm{x}$ & $\mathrm{x}$ & $\mathrm{x}$ \\
\hline \multicolumn{8}{|l|}{ Comesomatidae Filipjev, 1918 (16) } \\
\hline Comesoma sp. & - & $\mathrm{x}$ & $\mathrm{x}$ & - & - & - & - \\
\hline Dorylaimopsis punctata Ditlevsen, 1918 & - & $\mathrm{x}$ & $\mathrm{x}$ & $\mathrm{x}$ & $\mathrm{x}$ & $\mathrm{x}$ & - \\
\hline Dorylaimopsis sp. & - & $\mathrm{x}$ & $\mathrm{x}$ & $\mathrm{x}$ & $\mathrm{x}$ & $\mathrm{x}$ & $\mathrm{x}$ \\
\hline Laimella sp. & - & - & - & - & $\mathrm{x}$ & - & $\mathrm{x}$ \\
\hline Metacomesoma sp. & - & $\mathrm{x}$ & - & - & - & - & - \\
\hline Paracomesoma dubuim (Filipjev, 1918) & - & - & - & $\mathrm{x}$ & - & - & - \\
\hline Paracomesoma sp. & CMLRE 4/066 & $\mathrm{x}$ & $\mathrm{x}$ & - & $\mathrm{x}$ & $\mathrm{x}$ & $\mathrm{x}$ \\
\hline Sabatieria breviseta Stekhoven, 1935 & - & $\mathrm{x}$ & $\mathrm{x}$ & $\mathrm{x}$ & $\mathrm{x}$ & $\mathrm{x}$ & - \\
\hline Sabatieria celtica Southern, 1914 & CMLRE $4 / 030$ & $\mathrm{x}$ & $\mathrm{x}$ & $\mathrm{x}$ & $\mathrm{x}$ & - & - \\
\hline Sabatieria longisetosa (Kries, 1929) & - & $\mathrm{x}$ & - & $\mathrm{x}$ & - & $\mathrm{x}$ & - \\
\hline Sabatieria ornata (Ditlevsen, 1918) & - & $\mathrm{x}$ & $\mathrm{x}$ & $\mathrm{x}$ & - & $\mathrm{x}$ & - \\
\hline Sabatieria praedatrix De Man, 1907 & CMLRE $4 / 031$ & $\mathrm{x}$ & $\mathrm{x}$ & - & $\mathrm{x}$ & - & $\mathrm{x}$ \\
\hline Sabatieria pulchra (Schneider, 1906) & CMLRE 4/032 & $\mathrm{x}$ & $\mathrm{x}$ & $\mathrm{x}$ & $\mathrm{x}$ & $\mathrm{x}$ & $\mathrm{x}$ \\
\hline Sabatieria punctata (Kreis, 1924) & CMLRE $4 / 033$ & $\mathrm{x}$ & $\mathrm{x}$ & $\mathrm{x}$ & $\mathrm{x}$ & $\mathrm{x}$ & - \\
\hline Sabatieria sp.1 & - & $\mathrm{x}$ & $\mathrm{x}$ & $\mathrm{x}$ & $\mathrm{x}$ & $\mathrm{x}$ & $\mathrm{x}$ \\
\hline Sabatieria sp.2 & - & $\mathrm{x}$ & $\mathrm{x}$ & $\mathrm{x}$ & $\mathrm{x}$ & - & - \\
\hline \multicolumn{8}{|l|}{ Ethmolaimidae Filipjev and Stekhoven, 1941 (2) } \\
\hline Comesa cuanensis (Platt, 1982) & CMLRE 4/017 & $\mathrm{x}$ & $\mathrm{x}$ & - & - & - & - \\
\hline Paraethmolaimus sp. & - & $\mathrm{x}$ & - & - & - & - & - \\
\hline \multicolumn{8}{|l|}{ Cyatholaimidae Filipjev, 1918 (10) } \\
\hline Cyatholaimus sp. & CMLRE 4/065 & $\mathrm{x}$ & - & - & $\mathrm{x}$ & - & - \\
\hline Longicyatholaimus sp. & - & $\mathrm{x}$ & $\mathrm{x}$ & $\mathrm{x}$ & $\mathrm{x}$ & - & $\mathrm{x}$ \\
\hline Metacyatholaimus sp. & - & - & - & - & $\mathrm{x}$ & - & - \\
\hline Paracanthonchus longicaudatus Warwick, 1971 & CMLRE 4/072 & $\mathrm{x}$ & - & - & $\mathrm{x}$ & - & - \\
\hline Paracanthonchus platti Vadhyar, 1980 & - & - & $\mathrm{x}$ & - & - & - & - \\
\hline Paracanthonchus sp. & - & $\mathrm{x}$ & - & - & $\mathrm{x}$ & $\mathrm{x}$ & $\mathrm{x}$ \\
\hline Paracyatholaimus sp. & CMLRE 4/071 & - & - & $\mathrm{x}$ & $\mathrm{x}$ & - & - \\
\hline Paralongicyatholaimus minutus Warwick, 1971 & - & $\mathrm{x}$ & $\mathrm{x}$ & $\mathrm{x}$ & $\mathrm{x}$ & $\mathrm{x}$ & $\mathrm{x}$ \\
\hline Paralongicyatholaimus sp. & CMLRE $4 / 040$ & $\mathrm{x}$ & $\mathrm{x}$ & $\mathrm{x}$ & $\mathrm{x}$ & $\mathrm{x}$ & $\mathrm{x}$ \\
\hline Pomponema sp. & - & $\mathrm{x}$ & $\mathrm{x}$ & - & $\mathrm{x}$ & - & - \\
\hline \multicolumn{8}{|l|}{ Selachinematidae Cobb, 1915 (5) } \\
\hline Cheironchus sp. & - & $\mathrm{x}$ & - & - & - & - & - \\
\hline Gammanema sp. & - & $\mathrm{x}$ & - & - & - & - & - \\
\hline Halichoanolaimus robustus (Bastian, 1865) & - & - & - & - & $\mathrm{x}$ & - & - \\
\hline Synonchiella sp. & CMLRE 4/069 & - & $\mathrm{x}$ & - & - & - & $\mathrm{x}$ \\
\hline Synonchium sp. & - & - & $\mathrm{x}$ & - & - & - & - \\
\hline \multicolumn{8}{|l|}{ Desmodoridae Filipjev, 1922 (16) } \\
\hline Catanema sp. & - & - & $\mathrm{x}$ & - & - & - & - \\
\hline Chromaspirina inglisi Warwick, 1970 & CMLRE $4 / 014$ & $\mathrm{x}$ & - & - & - & - & - \\
\hline Chromaspirina parapontica Luc and De Coninck, 1959 & CMLRE 4/015 & $\mathrm{x}$ & - & - & $\mathrm{x}$ & - & - \\
\hline Chromaspirina sp. & - & $\mathrm{x}$ & - & $\mathrm{x}$ & $\mathrm{x}$ & - & - \\
\hline Desmodora (Desmodora) scaldensis De Man, 1889 & - & $\mathrm{x}$ & - & $\mathrm{x}$ & - & $\mathrm{x}$ & - \\
\hline Desmodora (Desmodora) schulzi Gerlach, 1950 & CMLRE $4 / 025$ & - & - & $\mathrm{x}$ & - & $\mathrm{x}$ & - \\
\hline Echinodesmadora sp. & - & $\mathrm{x}$ & - & - & $\mathrm{x}$ & - & - \\
\hline Leptonemella sp. & - & - & - & - & $\mathrm{x}$ & - & - \\
\hline Metachromadora (Bradylaimus) suecica (Allgen, 1929) & CMLRE 4/056 & $\mathrm{x}$ & - & - & - & - & - \\
\hline Metachromadora (Chromadoropsis) vivipara (De Man,1907) & - & $\mathrm{x}$ & - & - & - & - & - \\
\hline Metachromadora sp.1 & - & $\mathrm{x}$ & - & $\mathrm{x}$ & $\mathrm{x}$ & $\mathrm{x}$ & - \\
\hline Metachromadora sp.2 & CMLRE 4/055 & $\mathrm{x}$ & $\mathrm{x}$ & $\mathrm{x}$ & $\mathrm{x}$ & $\mathrm{x}$ & $\mathrm{x}$ \\
\hline Paradesmodora sp. & CMLRE $4 / 047$ & $\mathrm{x}$ & $\mathrm{x}$ & - & $\mathrm{x}$ & $\mathrm{x}$ & - \\
\hline Pseudonchus northumbriensis Warwick, 1969 & CMLRE $4 / 053$ & - & $\mathrm{x}$ & - & $\mathrm{x}$ & - & - \\
\hline Pseudonchus sp. & CMLRE $4 / 052$ & $\mathrm{x}$ & $\mathrm{x}$ & $\mathrm{x}$ & $\mathrm{x}$ & - & $\mathrm{x}$ \\
\hline Spirinia sp. & - & $\mathrm{x}$ & $\mathrm{x}$ & $\mathrm{x}$ & $\mathrm{x}$ & $\mathrm{x}$ & - \\
\hline \multicolumn{8}{|l|}{ Draconematidae Filipjev, 1918 (2) } \\
\hline Draconema claparedii (Gerlach, 1952) & - & $\mathrm{x}$ & - & - & - & - & - \\
\hline Draconema sp. & - & - & - & $\mathrm{x}$ & - & - & - \\
\hline \multicolumn{8}{|l|}{ Epsilonematidae Steiner, 1927 (1) } \\
\hline Epsilonema pustulatum (Gerlach, 1952) & CMLRE 4/077 & $\mathrm{x}$ & - & - & - & - & - \\
\hline
\end{tabular}


TABLE 1. Continued.

\begin{tabular}{|c|c|c|c|c|c|c|c|}
\hline NEMATODES & CMLRE NUMBER & 1 & 2 & 3 & 4 & 5 & 6 \\
\hline \multicolumn{8}{|l|}{ Microlaimidae Micoletzky, 1922 (11) } \\
\hline Aponema sp. & - & $\mathrm{x}$ & $\mathrm{x}$ & - & $\mathrm{x}$ & - & - \\
\hline Belbolaimus teutonicus Riemann, 1966 & - & $\mathrm{x}$ & $\mathrm{x}$ & - & - & - & - \\
\hline Belbolaimus sp. & CMLRE 4/041 & $\mathrm{x}$ & $\mathrm{x}$ & - & $\mathrm{x}$ & - & - \\
\hline Calomicrolaimus honestus (De Man, 1922) & CMLRE 4/011 & - & - & - & $\mathrm{x}$ & - & - \\
\hline Calomicrolaimus spirifer (Warwick, 1970) & - & $\mathrm{x}$ & - & $\mathrm{x}$ & $\mathrm{x}$ & - & - \\
\hline Calomicrolaimus sp. & CMLRE 4/012 & $\mathrm{x}$ & - & $\mathrm{x}$ & $\mathrm{x}$ & - & - \\
\hline Microlaimus acinaces Warwick and Platt, 1971 & - & - & $\mathrm{x}$ & - & - & - & - \\
\hline Microlaimus conothelis (Lorenzen, 1973a) & CMLRE 4/027 & $\mathrm{x}$ & - & - & - & - & - \\
\hline Microlaimus robustidens Stekhoven and De Connick, 1933 & CMLRE 4/026 & $\mathrm{x}$ & - & $\mathrm{x}$ & $\mathrm{x}$ & $\mathrm{x}$ & - \\
\hline Microlaimus sp.1 & - & $\mathrm{x}$ & $\mathrm{x}$ & $\mathrm{x}$ & $\mathrm{x}$ & $\mathrm{x}$ & $\mathrm{x}$ \\
\hline Microlaimus sp.2 & CMLRE 4/028 & $\mathrm{x}$ & $\mathrm{x}$ & - & $\mathrm{x}$ & - & - \\
\hline \multicolumn{8}{|l|}{ Leptolaimidae Orley, 1880 (10) } \\
\hline Camacolaimus barbatus Warwick, 1970 & - & $\mathrm{x}$ & - & - & - & - & - \\
\hline Camacolaimus langicauda De Man, 1922 & - & $\mathrm{x}$ & - & - & $\mathrm{x}$ & - & - \\
\hline Camacolaimus sp. & CMLRE 4/070 & $\mathrm{x}$ & $\mathrm{x}$ & $\mathrm{x}$ & $\mathrm{x}$ & $\mathrm{x}$ & - \\
\hline Deontolaimus sp. & - & - & $\mathrm{x}$ & - & - & - & $\mathrm{x}$ \\
\hline Leptolaimus ampullaceus Warwick, 1970 & CMLRE $4 / 062$ & $\mathrm{x}$ & - & - & - & $\mathrm{x}$ & - \\
\hline Laptolaimus elegans (Stekhoven and De Coninck, 1933) & - & $\mathrm{x}$ & - & - & $\mathrm{x}$ & $\mathrm{x}$ & - \\
\hline Leptolaimus papillinger De Man, 1876 & CMLRE 4/063 & $\mathrm{x}$ & - & - & - & - & - \\
\hline Leptolaimus sp. & - & $\mathrm{x}$ & - & - & $\mathrm{x}$ & - & - \\
\hline Leptolaimoides sp. & - & $\mathrm{x}$ & - & - & - & - & - \\
\hline Stephanolaimus sp. & - & $\mathrm{x}$ & - & - & - & - & - \\
\hline \multicolumn{8}{|l|}{ Ceramonematidae Cobb, 1933 (4) } \\
\hline Dasynemoides albaensis (Warwick and Platt, 1973) & - & - & - & $\mathrm{x}$ & - & - & - \\
\hline Metadasynemoides sp. & CMLRE 4/075 & $\mathrm{x}$ & - & $\mathrm{x}$ & $\mathrm{x}$ & $\mathrm{x}$ & $\mathrm{x}$ \\
\hline Pselionema longiseta Ward, 1974 & - & $\mathrm{x}$ & $\mathrm{x}$ & - & $\mathrm{x}$ & $\mathrm{x}$ & - \\
\hline Pselionema sp. & - & $\mathrm{x}$ & $\mathrm{x}$ & - & $\mathrm{x}$ & $\mathrm{x}$ & - \\
\hline \multicolumn{8}{|l|}{ Meyliidae De Coninck, 1965 (1) } \\
\hline Gerlachius sp. & - & - & $\mathrm{x}$ & - & - & - & - \\
\hline \multicolumn{8}{|l|}{ Desmoscolecidae Shipley, 1896 (6) } \\
\hline Desmoscolex falcatus Lorenzen, 1972 & - & $\mathrm{x}$ & - & - & - & - & - \\
\hline Desmoscolex sp. & - & $\mathrm{x}$ & - & $\mathrm{x}$ & - & - & - \\
\hline Quadricoma scanica (Allgen, 1935) & - & $\mathrm{x}$ & - & - & - & - & - \\
\hline Tricoma brevirostris (Southern, 1914) & - & $\mathrm{x}$ & $\mathrm{x}$ & $\mathrm{x}$ & $\mathrm{x}$ & $\mathrm{x}$ & $\mathrm{x}$ \\
\hline Tricoma longirostris (Southern, 1914) & CMLRE $4 / 074$ & $\mathrm{x}$ & $\mathrm{x}$ & $\mathrm{x}$ & $\mathrm{x}$ & $\mathrm{x}$ & $\mathrm{x}$ \\
\hline Tricoma sp. & - & $\mathrm{x}$ & $\mathrm{x}$ & $\mathrm{x}$ & $\mathrm{x}$ & $\mathrm{x}$ & $\mathrm{x}$ \\
\hline \multicolumn{8}{|l|}{ Aegialoalaimidae Lorenzen, 1981 (1) } \\
\hline Southernia sp. & - & - & $\mathrm{x}$ & $\mathrm{x}$ & $\mathrm{x}$ & - & - \\
\hline \multicolumn{8}{|l|}{ Monhysteridae De Man, 1876 (8) } \\
\hline Diplolaimelloides sp. & - & $\mathrm{x}$ & - & - & - & - & - \\
\hline Paramonhystera buetschlii (Bresslau and Stekhoven 1935) & - & - & - & $\mathrm{x}$ & $\mathrm{x}$ & - & $\mathrm{x}$ \\
\hline Paramonhystera riemanni (Platt, 1973) & - & - & - & $\mathrm{x}$ & - & - & - \\
\hline Paramonhystera sp.1 & - & $\mathrm{x}$ & $\mathrm{x}$ & $\mathrm{x}$ & $\mathrm{x}$ & $\mathrm{x}$ & $\mathrm{x}$ \\
\hline Paramonhystera sp.2 & CMLRE 4/029 & - & - & $\mathrm{x}$ & $\mathrm{x}$ & - & $\mathrm{x}$ \\
\hline Thalassomonhystera parva (Bastian, 1865) & - & $\mathrm{x}$ & - & $\mathrm{x}$ & - & $\mathrm{x}$ & $\mathrm{x}$ \\
\hline Thalassomonhystera venusta (Lorenzen, 1979) & - & $\mathrm{x}$ & $\mathrm{x}$ & $\mathrm{x}$ & $\mathrm{x}$ & $\mathrm{x}$ & $\mathrm{x}$ \\
\hline Thalassomonhystera sp. & CMLRE 4/067 & $\mathrm{x}$ & $\mathrm{x}$ & $\mathrm{x}$ & $\mathrm{x}$ & $\mathrm{x}$ & $\mathrm{x}$ \\
\hline \multicolumn{8}{|l|}{ Xyalidae Chitwood, 1951 (18) } \\
\hline Cobbia trefusiaeformis De Man, 1907 & CMLRE 4/016 & $\mathrm{x}$ & - & - & - & - & - \\
\hline Cobbia sp. & - & - & - & $\mathrm{x}$ & - & - & - \\
\hline Daptonema biggi (Gerlach, 1965) & CMLRE 4/020 & - & - & $\mathrm{x}$ & - & - & - \\
\hline Daptonema hirsutum (Vitiello, 1967) & CMLRE 4/018 & - & - & - & - & $\mathrm{x}$ & - \\
\hline Daptonema normandicum (De Man, 1890) & CMLRE 4/019 & $\mathrm{x}$ & $\mathrm{x}$ & $\mathrm{x}$ & $\mathrm{x}$ & $\mathrm{x}$ & $\mathrm{x}$ \\
\hline Daptonema oxycerca (De Man, 1888) & - & $\mathrm{x}$ & $\mathrm{x}$ & $\mathrm{x}$ & $\mathrm{x}$ & $\mathrm{x}$ & - \\
\hline Daptonema procerum (Gerlach, 1951) & - & - & - & $\mathrm{x}$ & - & - & - \\
\hline Daptonema psammoides (Warwick, 1970) & CMLRE 4/021 & - & $\mathrm{x}$ & $\mathrm{x}$ & - & - & - \\
\hline Daptonema setifer (Gerlach, 1952) & - & $\mathrm{x}$ & - & - & - & - & - \\
\hline Daptonema tenuispiculum (Ditlevsen, 1918) & CMLRE 4/022 & $\mathrm{x}$ & $\mathrm{x}$ & $\mathrm{x}$ & $\mathrm{x}$ & $\mathrm{x}$ & $\mathrm{x}$ \\
\hline Daptonema sp.1 & - & $\mathrm{x}$ & $\mathrm{x}$ & $\mathrm{x}$ & $\mathrm{x}$ & $\mathrm{x}$ & $\mathrm{x}$ \\
\hline Daptonema sp.2 & CMLRE 4/023 & $\mathrm{x}$ & $\mathrm{x}$ & $\mathrm{x}$ & $\mathrm{x}$ & $\mathrm{x}$ & $\mathrm{x}$ \\
\hline
\end{tabular}


TABle 1. Continued.

\begin{tabular}{|c|c|c|c|c|c|c|c|}
\hline NEMATODES & CMLRE NUMBER & 1 & 2 & 3 & 4 & 5 & 6 \\
\hline Daptonema sp.3 & CMLRE $4 / 024$ & - & $\mathrm{x}$ & $\mathrm{x}$ & $\mathrm{x}$ & $\mathrm{x}$ & $\mathrm{x}$ \\
\hline Echinotheristus sp. & - & - & - & - & $\mathrm{x}$ & - & - \\
\hline Theristus longus Platt, 1973 & CMLRE 4/064 & $\mathrm{x}$ & $\mathrm{x}$ & $\mathrm{x}$ & $\mathrm{x}$ & - & - \\
\hline Theristus ensifer Gerlach, 1951 & CMLRE $4 / 080$ & $\mathrm{x}$ & - & $\mathrm{x}$ & - & - & - \\
\hline Theristus sp.1 & - & $\mathrm{x}$ & $\mathrm{x}$ & $\mathrm{x}$ & $\mathrm{x}$ & - & - \\
\hline Theristus sp. 2 & - & - & - & $\mathrm{x}$ & - & - & - \\
\hline Sphaerolaimus balticus Schneider, 1906 & CMLRE 4/035 & - & $\mathrm{x}$ & - & $\mathrm{x}$ & $\mathrm{x}$ & - \\
\hline Sphaerolaimus gracilis De man, 1884 & CMLRE 4/036 & $\mathrm{x}$ & - & - & $\mathrm{x}$ & - & - \\
\hline Sphaerolaimus islandicus Ditlevsen, 1926 & - & $\mathrm{x}$ & $\mathrm{x}$ & $\mathrm{x}$ & $\mathrm{x}$ & $\mathrm{x}$ & $\mathrm{x}$ \\
\hline Sphaerolaimus sp. & - & $\mathrm{x}$ & $\mathrm{x}$ & - & - & $\mathrm{x}$ & - \\
\hline \multicolumn{8}{|l|}{ Siphonolaimidae Filipjev, 1918 (4) } \\
\hline Astomonema southwardorum Austen, Warwick and Ryan, 1993 & CMLRE 4/009 & $\mathrm{x}$ & - & $\mathrm{x}$ & $\mathrm{x}$ & - & - \\
\hline Siphonolaimus cobbi Riemann, 1966 & CMLRE $4 / 034$ & - & - & $\mathrm{x}$ & $\mathrm{x}$ & - & - \\
\hline Siphonolaimus sp. & - & $\mathrm{x}$ & - & - & - & - & - \\
\hline \multicolumn{8}{|l|}{ Linhomoeidae Filipjev, 1922 (15) } \\
\hline Eleutherolaimus sp. & - & $\mathrm{x}$ & - & - & - & - & - \\
\hline Linhomoeus hirsutus Bastian, 1865 & - & - & - & - & $\mathrm{x}$ & - & - \\
\hline Linhomoeus sp.1 & - & $\mathrm{x}$ & - & $\mathrm{x}$ & $\mathrm{x}$ & $\mathrm{x}$ & - \\
\hline Linhomoeus sp.2 & - & $\mathrm{x}$ & $\mathrm{x}$ & - & - & - & - \\
\hline Megadesmolaimus sp. & CMLRE $4 / 054$ & $\mathrm{x}$ & - & - & $\mathrm{x}$ & - & - \\
\hline Metalinhomoeus filiformis (De Man, 1907) & CMLRE $4 / 042$ & $\mathrm{x}$ & $\mathrm{x}$ & $\mathrm{x}$ & $\mathrm{x}$ & $\mathrm{x}$ & - \\
\hline Metalinhomoeus longiseta Kreis, 1929 & - & - & $\mathrm{x}$ & - & $\mathrm{x}$ & $\mathrm{x}$ & - \\
\hline Metalinhomoeus sp. & - & $\mathrm{x}$ & $\mathrm{x}$ & $\mathrm{x}$ & $\mathrm{x}$ & $\mathrm{x}$ & $\mathrm{x}$ \\
\hline Paralinhomoeus conicaudatus (Allgen, 1930) & CMLRE $4 / 049$ & $\mathrm{x}$ & - & $\mathrm{x}$ & $\mathrm{x}$ & - & $\mathrm{x}$ \\
\hline Paralinhomoeus lepturus (De Man, 1907) & - & $\mathrm{x}$ & - & $\mathrm{x}$ & $\mathrm{x}$ & - & - \\
\hline Paralinhomoeus uniovarium Warwick, 1970 & CMLRE 4/048 & $\mathrm{x}$ & - & - & - & - & - \\
\hline Terschellingia communis De Man, 1888 & - & $\mathrm{x}$ & $\mathrm{x}$ & $\mathrm{x}$ & $\mathrm{x}$ & $\mathrm{x}$ & $\mathrm{x}$ \\
\hline Terschellingia longicaudata De Man, 1907 & - & $\mathrm{x}$ & $\mathrm{x}$ & $\mathrm{x}$ & $\mathrm{x}$ & $\mathrm{x}$ & $\mathrm{x}$ \\
\hline Terschellingia sp. & CMLRE 4/050 & $\mathrm{x}$ & $\mathrm{x}$ & $\mathrm{x}$ & $\mathrm{x}$ & $\mathrm{x}$ & $\mathrm{x}$ \\
\hline \multicolumn{8}{|l|}{ Axonolaimidae Filipjev, 1918 (11) } \\
\hline Ascolaimus elongates (Butschli, 1874) & - & $\mathrm{x}$ & - & - & $\mathrm{x}$ & - & - \\
\hline Ascolaimus sp. & CMLRE $4 / 051$ & $\mathrm{x}$ & $\mathrm{x}$ & $\mathrm{x}$ & $\mathrm{x}$ & $\mathrm{x}$ & $\mathrm{x}$ \\
\hline Axonolaimus paraspinosus Stekhoven and Adam, 1931 & CMLRE $4 / 010$ & $\mathrm{x}$ & - & - & - & $\mathrm{x}$ & - \\
\hline Axonolaimus sp. & - & $\mathrm{x}$ & - & - & $\mathrm{x}$ & $\mathrm{x}$ & - \\
\hline Odontophora exharena Warwick and Platt, 1973 & CMLRE $4 / 043$ & $\mathrm{x}$ & - & - & $\mathrm{x}$ & $\mathrm{x}$ & - \\
\hline Odontophora longisetosa (Allgen, 1928) & CMLRE $4 / 044$ & $\mathrm{x}$ & - & $\mathrm{x}$ & $\mathrm{x}$ & $\mathrm{x}$ & $\mathrm{x}$ \\
\hline Odontophora rectangula Lorenzen, 1971 & CMLRE $4 / 045$ & $\mathrm{x}$ & - & $\mathrm{x}$ & $\mathrm{x}$ & $\mathrm{x}$ & $\mathrm{x}$ \\
\hline Odontophora setosa (Allgen, 1929) & - & $\mathrm{x}$ & - & $\mathrm{x}$ & $\mathrm{x}$ & - & - \\
\hline Odontophora sp.1 & CMLRE $4 / 046$ & $\mathrm{x}$ & $\mathrm{x}$ & $\mathrm{x}$ & $\mathrm{x}$ & $\mathrm{x}$ & $\mathrm{x}$ \\
\hline Odontophora sp.2 & - & $\mathrm{x}$ & $\mathrm{x}$ & $\mathrm{x}$ & $\mathrm{x}$ & $\mathrm{x}$ & $\mathrm{x}$ \\
\hline Parodontophora sp. & - & - & - & $\mathrm{x}$ & - & - & - \\
\hline \multicolumn{8}{|l|}{ Diplopeltidae Filipjev, 1918 (3) } \\
\hline Araeolaimus elegans De Man, 1888 & CMLRE 4/007 & $\mathrm{x}$ & $\mathrm{x}$ & $\mathrm{x}$ & $\mathrm{x}$ & - & - \\
\hline Araeolaimus sp.1 & - & $\mathrm{x}$ & $\mathrm{x}$ & $\mathrm{x}$ & - & $\mathrm{x}$ & - \\
\hline Araeolaimus sp.2 & CMLRE 4/008 & $\mathrm{x}$ & $\mathrm{x}$ & - & $\mathrm{x}$ & $\mathrm{x}$ & - \\
\hline
\end{tabular}

ACKNOWLEDGMENTS: We would like to thank to an anonymous referee for comments which helped to improve the manuscript.

\section{Literature Cited}

Ansari, Z.A., A.H. Parulekar and T.G. Jagtap. 1980. Distribution of sublittoral meiobenthos off Goa coast, India. Hydrobiologia 74 (3): 209 214 .

Armenteros, M., J.A. Perez-Garcia, A. Perez-Angulo and J.P. Williams. 2008 Efficiency of extraction of meiofauna from sandy and muddy marine sediments. Revista de Investigaciones Marinas, 29: 113-118.

Armenteros, M., A. Ruiz-Abierno, R. Fernandez-Garces, J.A. Pe'ez-Garcia, L. Diaz-Asencio, M. Vincx and W. Decraemer. 2009. Biodiversity patterns of free-living marine nematodes in a tropical bay: Cienfuegos, Caribbean Sea. Esturine, Coastal and Shelf Science 85:179-189.
Bongers, T. and H. Ferris. 1999. Nematode community structure as a bioindicator in environmental monitoring. Trends in Ecology and Evolution 14: 224-228.

De Bovee, F., L.D. Guidi and J. Soyer. 1990. Quantitative distribution of deep sea meiobenthos in the northwestern Mediterranean (Gulf of Lions). Continental Shelf Research 10: 1123-1145.

De Leonardis, C., R. Sandulli, J. Vanaverbeke, M. Vincx and S. De Zio. 2008. Meiofauna and nematode diversity in some Mediterranean subtidal areas of the Adriatic and Ionian Sea. Scientia Marina, 72: 5-13.

Harkantra, S.N., A. Nair, Z.A. Ansari and A.H. Parulekar. 1980. Benthos of the shelf region along the west coast of India, Indian Journal of Marine Science 9:106-110.

Heip, C., M. Vincx and G. Vranken. 1985. The ecology of marine nematodes. Oceanography and Marine Biology Annual Review 23: 399-489. 
Higgins, R.P. and H. Thiel. 1988. Introduction to the study of meiofauna. Washington: Smithsonian Institution Press. 231p.

Liu, X. S., Z. N. Zhang and Y. Huang. 2007. Sublittoral meiofauna with particular reference to nematodes in the southern Yellow Sea, China. Estuarine, Coastal and Shelf Science 71: 616-628.

Muthumbi, A.W., A. Vanreusel, G. Duineveld, K. Soetaert and M. Vincx. 2004. Nematode community structure along the continental slope off the Kenyan coast, Western Indian Ocean. International Review of Hydrobiology 89: 188-205.

Parulekar, A. H., S. N. Harkantra and Z. A. Ansari. 1982. Benthic production and assessment of demersal fishery resources of the Indian Seas. Indian Journal of Marine Science 11: 107-111.

Platt, H.M. and R.M. Warwick. 1980. The significance of nematodes to the littoral ecosystem; p.729-759 In J.H. Prince, D.E.C. Irvine and W.H. Franham (ed.). The shore environment, ecosystems Volume II. London: Academic Press.

Platt, H. M. and R.M. Warwick. 1983. Free living marine nematodes, Part I, British Enoplids, Pictorial key to world genera and notes for the identification of British species; p.1-307 In D.M. Kermack and R.S.K. Barnes (ed.). Synopses of the British Fauna. Volume 28. Cambridge: Cambridge University Press.

Platt, H. M. and R.M. Warwick. 1988. Free living marine nematodes, Part II, British Chromodorids, Pictorial key to world genera and notes for the identification of British species; p. 1-502 In D.M. Kermack and R.S.K. Barnes (ed.). Synopses of the British Fauna (New series). Volume 38. Leiden: Brill/ Backhuys.

Sajan, S. and R. Damodaran. 2007. Faunal composition of meiobenthos from the shelf region off west coast of India, Journal of Marine Biological Association, India 49(1): 19-26.

Sajan, S., T.V. Joydas and R. Damodaran. 2010. Meiofauna of the western continental shelf of India, Arabian Sea. Estuarine, Coastal and Shelf Science 86 (4): 665-674.

Schratzberger, M., K.Warr and S.I.Rogers. 2007. Functional diversity of nematode communities in the southwestern North Sea. Marine Environmental Research 63: 368-389.

Snelgrove, V.R., T.H. Blackburn, P.A. Hutchings, D.M. Alongi, J.F. Grassle, H. Hummel, G. King, I. Koike, P.J.D. Lambshead, N.B. Ramsing and V. Solis-Weiss. 1997. The importance of marine sediment biodiversity in ecosystem processes. Ambio 26: 578-583.
Soetaert, K. and C. Heip. 1995. Nematode assemblage of deep sea and shelf break sites in the North Atlantic and Mediterranean Sea. Marine Ecological Progress Series 125: 171-183.

Soltwedel, T. 2000. Metazoan meiobenthos along continental margins: A Review. Progress in Oceanography 46: 59-84.

Steyaert, M., T. Deprez, M. Raes, T. Bezerra, I. Demesel, S. Derycke, G. Desmet, G. Fonseca, M. de Assunc ão Franco, T. Gheskiere, E. Hoste, J. Ingels, T. Moens, J. Vanaverbeke, S. VanGaever, S. Vanhove, A. Vanreusel, D. Verschelde and M. Vincx. 2005. Electronic Key to the free-living marine Nematodes. Electronic Database Accessible at http://nemys.ugent.be/. Captured on September 2005.

Tietjen, J.H. 1992. Abundance and biomass of metazoan meiobenthos in the deep sea; p.45-62 In G.Rowe and V. Pariente (ed.). Deep-sea food chain and the global carbon cycle. Volume 360. Dordrecht: Kluwer Academic Publishers.

Timm, R.W.1961. The marine nematodes of the Bay of Bengal. Proceeding of Pakistan Academy Science 1:1-84.

Timm, R.W. 1967. Some estuarine nematodes from the Sunderbans. Proceeding of Pakistan Academy Science 4: 1-14.

Vanreusel, A., M. Vincx, D. Van Ganskbeke and W. Gijselinck. 1992. Structural analysis of the meiobenthos communities of the shelf break area in two stations of the Gulf of Biscay (North East Atlantic). Belgian Journal of Zoology 122: 185-202.

Vincx, M. 1996. Meiofauna in marine and brackish water sediments; $p$. 187-195 In G.S. Hall (ed.). Methods for Examination of Organismal Diversity in Soils and Sediments, Cambridge: CAB International.

Vranken, G. and C. Heip. 1983. Calculation of the intrinsic rate of natural increase. $r_{m}$ with Rhabditis marina Bastian, 1865 (Nematoda). Nematologica, 29: 468-477.

Warwick, R. M., H.M. Platt and P.J.D. Somerfield. 1998. Free-living marine nematodes, Part III, Monhysterids, Pictorial key to world genera and notes for the identification of British species; p. 1-296 In R.S.K. Barnes and J.H. Crothers (ed.). Synopses of the British Fauna (New series). Volume 53. Shrewsbury: Field Studies Council.

RECEIVED: December 2011

ACCEPTED: March 2012

Published ONLINE: June 2012

EDITORIAL RESPONSIBILITY: Simone Chinicz Cohen 